\title{
Community-onset Klebsiella pneumoniae pneumonia in Taiwan: clinical features of the disease and associated microbiological characteristics of isolates from pneumonia and nasopharynx
}

\author{
Yi-Tsung Lin ${ }^{1,2}{ }^{*}$, Yu-Ping Wang ${ }^{1}$, Fu-Der Wang ${ }^{1,2}$ and Chang-Phone Fung ${ }^{1,2}$ \\ ${ }^{1}$ Division of Infectious Diseases, Department of Medicine, Taipei Veterans General Hospital, Taipei, Taiwan \\ ${ }^{2}$ School of Medicine, National Yang-Ming University, Taipei, Taiwan
}

\section{Edited by:}

Evangelos Giamarellos-Bourboulis, Medical School of University of Athens, Greece

\section{Reviewed by:}

Alan S. Cross, University of Maryland School of Medicine, USA George Dimopoulos, University Hospital Attikon, Greece

\section{*Correspondence:}

Yi-Tsung Lin, Division of Infectious Diseases, Department of Medicine, Taipei Veterans General Hospital, Number 201, Section 2, Shih-Pai Road, Beitou, Taipei 112, Taiwan e-mail:ytlin8@vghtpe.gov.tw

\begin{abstract}
Klebsiella pneumoniae is an important cause of community-onset pneumonia in Asian countries and South Africa. We investigated the clinical characteristics of $K$. pneumoniae causing community-onset pneumonia, and the associated microbiological features between $K$. pneumoniae isolates from pneumonia and those from the nasopharynx in Taiwan. This study was conducted at the Taipei Veterans General Hospital during July, 2012 to February, 2014. The clinical characteristics in patients with community-onset $K$. pneumoniae pneumonia were analyzed. K. pneumoniae isolates from the nasopharynx of adults attending otorhinolaryngology outpatient clinics were collected to compare their microbiological features with those from pneumonia. Capsular genotypes, antimicrobial susceptibility, and multilocus sequence type (MLST) were determined among these strains. Ninety-one patients with community-onset $K$. pneumoniae pneumonia were enrolled. We found a high mortality (29.7\%) among these patients. Capsular types K1, K2, K5, K20, K54, and $\mathrm{K} 57$ accounted for $\sim 70 \%$ of the K. pneumoniae isolates causing pneumonia, and $\sim 70 \%$ of all the K. pneumoniae strains isolated from the nasopharynx of patients in outpatient clinics. The MLST profiles further demonstrated the genetic relatedness between most pneumonia isolates and those from the nasopharynx. In conclusion, our results show that community-onset pneumonia caused by $K$. pneumoniae was associated with high mortality and could have a reservoir in the nasopharynx. To tackle this high-mortality disease, the distribution of capsular types in the nasopharynx might have implications for future vaccine development.
\end{abstract}

Keywords: capsular types, Klebsiella pneumoniae, MLST, nasopharynx, pneumonia

\section{INTRODUCTION}

Klebsiella pneumoniae is recognized 100 years ago as a potential cause of community-acquired pneumonia (CAP). However, over the past few decades, $K$. pneumoniae has been a rare cause of CAP in North America, Europe, and Australia (Shon et al., 2013). By contrast, it is an important cause of lower respiratory tract infection (LRTI) in Asian countries and South Africa and is associated with high mortality (Wang et al., 2005; Yu et al., 2007; Song et al., 2008; Lin et al., 2010a,b). In one prospective surveillance study from eight Asian countries, Streptococcus pneumoniae (29.2\%) was the most common bacterial pathogen, followed by K. pneumoniae (15.4\%; Song et al., 2008). In Cambodia, K. pneumoniae accounted for $8 \%$ of community-acquired LRTI (Rammaert et al., 2012). A previous study in an island in the Indian Ocean $2,500 \mathrm{~km}$ east of the South African coast demonstrated that $S$. pneumoniae and $K$. pneumoniae ( 42 and 22\%, respectively) were the most frequent microbial agents in CAP patients admitted to intensive care units (Paganin et al., 2004).

In Western countries, although K. pneumoniae is a rare cause of CAP, one recent study highlighted that CAP caused by $K$. pneumoniae was associated with increased risk of cardiovascular events following this infection (Griffin et al., 2013). Recently, the emergence of fatal bacteremic CAP due to K. pneumoniae was reported in France (Decre et al., 2011; Rafat et al., 2013). This suggests that the re-emergence of severe $K$. pneumoniae pulmonary infections in Western countries cannot be overlooked.

Klebsiella pneumoniae, especially the virulent capsular types $\mathrm{K} 1$ and $\mathrm{K} 2$, has emerged as the major cause of communityacquired liver abscess in the past three decades in East Asia, especially Taiwan (Fung et al., 2002; Fang et al., 2004; Lin et al., 2013). However, the clinical features and microbiological characteristics of $K$. pneumoniae causing community-onset pneumonia have rarely been addressed (Lin etal., 2010a,b). The pathogenesis of $K$. pneumoniae causing community-onset pneumonia is unknown and nasopharyngeal colonization may precede pneumonia.

In the current study, we investigated the clinical features of community-onset pneumonia caused by $K$. pneumoniae in our hospital. We further compared the microbiological characteristics between $K$. pneumoniae strains from the nasopharynx in adult patients attending otorhinolaryngology outpatient clinics and those from community-onset pneumonia. 


\section{MATERIALS AND METHODS STUDY DESIGN AND PATIENTS}

A retrospective study was carried out in the Taipei Veterans General Hospital from July, 2012 to February, 2014. All consecutive adult patients (aged $>20$ years) admitted with community-onset pneumonia due to $K$. pneumoniae were included. Communityonset pneumonia refers to both CAP and healthcare-associated pneumonia (HCAP). Polymicrobial infections were excluded in this study. CAP, HCAP, and hospital-acquired pneumonia were diagnosed based on the criteria previously described (Maruyama et al., 2013). HCAP included patients with pneumonia and any of the following: (1) hospitalization for 2 days during the preceding 90 days; (2) residence in a nursing home or extended care facility; (3) home infusion therapy (including antibiotics); (4) chronic dialysis (including haemodialysis and peritoneal dialysis) during the preceding 30 days; or (5) home wound care. Taiwan is endemic for K. pneumoniae liver abscess and cases of liver abscess with septic lung metastasis were also excluded.

We also consecutively collected $K$. pneumoniae isolates from the nasopharyngeal region in adult patients (aged $>20$ years) attending otorhinolaryngology outpatient clinics from July, 2013 to February, 2014 to determine their clinical and microbiological characteristics. The main diagnosis of patients attending otorhinolaryngology outpatient clinics during this study period were acute sinusitis, chronic sinusitis, and allergic rhinitis. The protocol was approved by the hospital's institutional review board.

\section{DATA COLLECTION AND OUTCOME MEASURES}

The following data were collected on admission to hospital: age, sex, comorbid illnesses, clinical symptoms, concomitant bacteremia, and antimicrobial treatment. The Pneumonia Severity Index (PSI) was determined in all patients (Fine et al., 1997). In this observational study, we focused on the following endpoints: 28-day and in-hospital mortality, receiving mechanical ventilation during hospitalization and requiring intensive care.

\section{MICROBIOLOGICAL EVALUATION}

The Vitek 2 automated system (bioMérieux, Marcy l'Etoile, France) was used for bacterial identification and antimicrobial susceptibility testing. The following antimicrobial agents were assayed: ampicillin, cefazolin, cefuroxime, cefoxitin, ceftriaxone, ceftazidime, cefepime, piperacillin-tazobactam, ertapenem, imipenem, amikacin, gentamicin, ciprofloxacin, levofloxacin, trimethoprim-sulfamethoxazole, and tigecycline. To determine the capsular genotypes of $K$. pneumoniae, we undertook cps genotyping by the polymerase chain reaction detection of $\mathrm{K}$ serotype-specific alleles at $w z y$ loci, including serotypes $\mathrm{K} 1, \mathrm{~K} 2$, $\mathrm{K} 5$, K20, K54, and K57, as described previously (Fang et al., 2007). Other capsular types were determined by a simple and useful capsular genotyping method for K. pneumoniae based on $w z c$ sequences as described previously (Pan et al., 2013). rmpA (regulator of the mucoid phenotype), a gene known as an extracapsular polysaccharide synthesis regulator, can positively control the mucoid phenotype of $K$. pneumoniae. The presence of $r m p A$ was proposed as an important virulence factor (Yu et al., 2006). One recent study showed that plasmid rmpA genes could be coinherited together with the adjacent virulence genes carried by a large plasmid in K. pneumoniae (Hsu et al., 2011). Plasmid rmpA and $r m p A 2$ genes were determined as described previously (Hsu et al., 2011).

Multi-locus sequence type (MLST) was performed on all isolates according to the protocol described on the K. pneumoniae MLST website (http://www.pasteur.fr/recherche/genopole/PF8/ $\mathrm{mlst} /$ ). MLST results were analyzed using the international K. pneumoniae MLST database created in 2005 at the Pasteur Institute in Paris, France (Diancourt et al., 2005).

\section{STATISTICAL ANALYSIS}

The $\chi^{2}$ test or Fisher's exact test were used in the comparison of categorical data, and the Student's $t$-test or Mann-Whitney $U$ test were used for the comparison of continuous variables. Univariate and multivariate logistic regression analyses were performed to predict 28-day mortality. Variables that showed a significant difference $(P<0.1)$ in the univariate analysis were included in the forward likelihood ratio, stepwise multivariate logistic regression model to determine if any of them were independently related to outcome. Analysis was performed using SPSS version 19.0 (SPSS Inc., Chicago, IL, USA) with $P<0.05$ considered statistically significant.

\section{RESULTS}

\section{CLINICAL CHARACTERISTICS OF PATIENTS WITH COMMUNITY-ONSET} K. pneumoniae PNEUMONIA AND RISK FACTORS FOR MORTALITY

During the study period, a total of 191 consecutive patients with monomicrobial $K$. pneumoniae pneumonia were identified. Of these patients, $91(47.6 \%)$ were classified as having communityonset infection, and the remaining 100 (52.6\%) were classified as having hospital-acquired infection. Among the 91 patients diagnosed with community-onset $K$. pneumoniae pneumonia, there was a male predominance $(n=76)$ and the mean age was $77.9 \pm 11.7$ years. CAP accounted for $51.6 \%(n=47)$ of the patients. None of the cases had concomitant distant abscesses. The 28-day mortality was $29.7 \%$ (27 patients). The overall in-hospital mortality was $34.1 \%$ (31 patients). Table 1 lists the clinical features of CAP and HCAP. HCAP patients had a significantly higher Charlson Comorbidity Index and PSI scores than CAP patients. CAP patients had a higher level of appropriate antibiotic use. Both the 28-day mortality and in-hospital mortality were not different statistically between CAP and HCAP.

\section{MICROBIOLOGICAL CHARACTERISTICS OF $K$. pneumoniae FROM PATIENTS WITH COMMUNITY-ONSET PNEUMONIA}

Table 2 shows the distribution of capsular type among $K$. pneumoniae isolates. Capsular types $\mathrm{K} 1, \mathrm{~K} 2, \mathrm{~K} 5, \mathrm{~K} 20$, K54, and $\mathrm{K} 57$ accounted for $67 \%(61 / 91)$ of all the K. pneumoniae isolates. During the same collection period for K. pneumoniae isolates from outpatients, the rate of capsular types $\mathrm{K} 1, \mathrm{~K} 2, \mathrm{~K} 5, \mathrm{~K} 20, \mathrm{~K} 54$, and K57 was consistent $(72 \%, 31 / 43)$. Plasmid $r m p A$ and $r m p A 2$ genes were identified in 65 and 63 isolates, respectively. All but two strains belonging to the six capsular types (K1, K2, K5, K20, K54, and K57) carried the plasmid rmpA or rmpA2 genes. Seventy-one of the $91(78 \%)$ isolates had the wild-type antibiotic susceptibility (susceptible to several classes of antibiotics, except for intrinsic resistance to ampicillin). MLST profiles were further determined 
Table 1 | Clinical features of CAP versus HCAP caused by Klebsiella pneumoniae.

\begin{tabular}{|c|c|c|c|}
\hline Characteristic & CAP $(n=47)$ & HCAP $(n=44)$ & $P$-value \\
\hline Age (year), mean $\pm S D$ & $76.9 \pm 12.2$ & $80.0 \pm 11.1$ & 0.387 \\
\hline \multicolumn{4}{|l|}{ Underlying diseases } \\
\hline Diabetes mellitus & $21(44.7)$ & $21(47.7)$ & 0.771 \\
\hline Malignancy & $13(27.7)$ & $16(36.4)$ & 0.373 \\
\hline Chronic kidney disease stage $\geq 4$ & $4(8.5)$ & $9(20.5)$ & 0.104 \\
\hline Chronic lung diseases & 15 (31.9) & $10(22.7)$ & 0.326 \\
\hline Liver cirrhosis & $2(6.7)$ & $1(3.6)$ & 1.000 \\
\hline Alcoholism & $4(8.5)$ & $3(6.8)$ & 1.000 \\
\hline PSI, class IV and V & $41(87.2)$ & $41(93.2)$ & 0.487 \\
\hline PSI, class V & $29(61.7)$ & $31(70.5)$ & 0.379 \\
\hline Days of hospitalization & $23.3 \pm 20.2$ & $21.8 \pm 17.7$ & 0.695 \\
\hline Initial presentation with septic shock & $22(46.8)$ & $13(29.5)$ & 0.091 \\
\hline Initial presentation with respiratory failure & $23(48.9)$ & $23(52.3)$ & 0.750 \\
\hline Requirement of mechanical ventilation during the course & $18(38.3)$ & $21(47.7)$ & 0.364 \\
\hline Intensive care unit admission & $30(63.8)$ & $26(59.1)$ & 0.642 \\
\hline Complicated with bacteraemia & $12(25.5)$ & $12(27.3)$ & 0.851 \\
\hline Appropriate antibiotics & $45(95.7)$ & $35(79.5)$ & 0.018 \\
\hline
\end{tabular}

Values given as mean $\pm S D$ or number of patients (\%).

in the isolates with capsular types $\mathrm{K} 1, \mathrm{~K} 2, \mathrm{~K} 5$, K20, K54, and K57 (Table 3).

\section{CAPSULAR TYPE OF $K$. pneumoniae ISOLATED FROM NASOPHARYNX IN OUTPATIENTS}

Seventy-one isolates from the nasopharyngeal region collected consecutively in otorhinolaryngology outpatient clinics were available for capsular genotyping. Of the 71 patients ( 45 were male), the mean age was $55.3 \pm 15.6$ years. Most patients $(57 / 71,80.2 \%)$ were healthy and no underlying diseases were documented. Diabetes mellitus $(6 / 14,42.9 \%)$ and malignancy $(6 / 14,42.9 \%)$ were the most common underlying diseases among the other 14 patients. Most of them did not receive any antibiotic in the visit. K. pneumoniae accounted for $11.5 \%$ (39/340) of all bacteria isolated from nasopharyngeal region from November, 2013 to February, 2014.

The detailed capsular types are shown in Table 2. Capsular types $\mathrm{K} 1, \mathrm{~K} 2$, K5, K20, K54, and $\mathrm{K} 57$ accounted for $\sim 70 \%(n=49)$ of all the K. pneumoniae isolates. Plasmid rmpA and rmpA2 genes were identified in 55 and 52 isolates, respectively. All K. pneumoniae isolates that belonged to these six capsular types carried plasmid rmpA or rmpA2 genes. Sixty-eight K. pneumoniae isolates showed the wild-type antibiotic susceptibility. Among the other three isolates, one showed an extended-spectrum $\beta$-lactamase phenotype, and the other two showed resistance to fluoroquinolones. The detailed MLST profiles in the capsular types K1, K2, K5, K20, $\mathrm{K} 54$, and K57 isolates are shown in Table 3. ST23, ST65, ST86, ST373, ST375, ST705, ST268, ST29, ST218, and ST592 were found in isolates from both pneumonia and the nasopharynx.

\section{COMPARISON OF MICROBIOLOGICAL CHARACTERISTICS AMONG $\boldsymbol{K}$. pneumoniae ISOLATES COLLECTED FROM PATIENTS WITH COMMUNITY-ONSET PNEUMONIA AND OUTPATIENTS}

Regarding the microbiological characteristics (Table 4), the proportion of capsular type K1, K2, K5, K20, K54, and $\mathrm{K} 57$, wild-type antibiotic susceptibility and presence of plasmid $r m p A / A 2$ were significantly higher in isolates from CAP than those from HCAP. In other words, $8.5 \%$ of CAP, $36.4 \%$ of HCAP, and $4.2 \%$ of outpatients isolates showed multidrug resistance. The characteristics were similar between isolates from CAP and outpatients. However, the proportion of wild-type antibiotic susceptibility and presence of plasmid $r m p A / A 2$ were significantly higher in isolates from outpatients than those from HCAP. 
Table 2 | Capsular type distribution among $K$. pneumoniae isolates collected from patients with community-onset pneumonia and nasopharynx from outpatients.

\begin{tabular}{llll}
\hline $\begin{array}{l}\text { Capsular } \\
\text { type }\end{array}$ & CAP isolates & HCAP isolates & Nasopharynx from \\
& No. $(n=47)$ & No. $(n=44)$ & $\begin{array}{l}\text { outpatients No. } \\
(n=71)\end{array}$
\end{tabular}

\begin{tabular}{|c|c|c|c|}
\hline K1 & 13 & 9 & 14 \\
\hline K2 & 12 & 9 & 20 \\
\hline K3 & 0 & 1 & 1 \\
\hline K5 & 2 & 1 & 2 \\
\hline K10 & 0 & 1 & 0 \\
\hline K16 & 3 & 0 & 2 \\
\hline K20 & 3 & 2 & 5 \\
\hline K21 & 1 & 0 & 0 \\
\hline K22 & 0 & 1 & 0 \\
\hline K25 & 0 & 0 & 1 \\
\hline K28 & 0 & 1 & 0 \\
\hline K37 & 0 & 1 & 0 \\
\hline K39 & 0 & 0 & 1 \\
\hline K52 & 0 & 0 & 3 \\
\hline K54 & 3 & 2 & 3 \\
\hline K57 & 3 & 2 & 5 \\
\hline K58 & 0 & 1 & 0 \\
\hline K61 & 0 & 1 & 0 \\
\hline K62 & 2 & 2 & 6 \\
\hline K64 & 0 & 3 & 4 \\
\hline K68 & 1 & 0 & 0 \\
\hline K74 & 0 & 1 & 0 \\
\hline K79 & 1 & 1 & 0 \\
\hline Others ${ }^{a}$ & 3 & 5 & 4 \\
\hline
\end{tabular}

a Other capsular types included KN2 $(n=3)$, probable new capsular types $(n=4)$ and non-typeable $(n=1)$.

\section{RISK FACTORS FOR MORTALITY}

Univariate analyses of the factors associated with 28-day mortality are shown in Table 5. The microbiological characteristics of K. pneumoniae were not associated with mortality. The logistic regression model showed that malignancy [odds ratio (OR) 4.08, 95\% confidence interval (CI) $1.32-12.66, P=0.015)$ and PSI scores (OR 1.017, 95\% CI 1.001-1.034, $P=0.042$ ) were independent risk factors for 28-day mortality. Initial presentation with respiratory failure (OR 3.38, 95\% CI 0.99-11.44, $P=0.051$ ) showed borderline significance in predicting mortality.

\section{DISCUSSION}

The present study demonstrated the clinical features of community-onset $K$. pneumoniae pneumonia as well as the distribution of capsular types and MLST of K. pneumoniae in a medical center in Taiwan. We found a high mortality (29.7\%) among these patients. Capsular types K1, K2, K5, K20, K54, and K57 accounted for $\sim 70 \%$ of all the K. pneumoniae isolates from
Table 3 | MLST profiles among $K$. pneumoniae isolates collected from patients with community-onset pneumonia and nasopharynx from outpatients.

\begin{tabular}{|c|c|c|c|}
\hline $\begin{array}{l}\text { Capsular type and } \\
\text { MLST profiles }\end{array}$ & $\begin{array}{l}\text { CAP No. } \\
(n=36)\end{array}$ & $\begin{array}{l}\text { HCAP No. } \\
(n=25)\end{array}$ & $\begin{array}{l}\text { Nasopharynx from } \\
\text { outpatients No. } \\
(n=49)\end{array}$ \\
\hline K1 & 13 & 9 & 14 \\
\hline ST23 & 13 & 9 & 13 \\
\hline ST260 & 0 & 0 & 1 \\
\hline K2 & 12 & 9 & 20 \\
\hline ST65 & 4 & 3 & 8 \\
\hline ST86 & 5 & 2 & 9 \\
\hline ST373 & 1 & 2 & 2 \\
\hline ST375 & 2 & 2 & 1 \\
\hline K5 & 2 & 1 & 2 \\
\hline ST76 & 1 & 0 & 0 \\
\hline ST660 & 1 & 0 & 0 \\
\hline ST705 & 0 & 1 & 0 \\
\hline ST1049 & 0 & 0 & 2 \\
\hline K20 & 3 & 2 & 5 \\
\hline ST268 & 2 & 1 & 2 \\
\hline ST420 & 1 & 1 & 0 \\
\hline ST1544 & 0 & 0 & 3 \\
\hline K54 & 3 & 2 & 3 \\
\hline ST29 & 3 & 1 & 2 \\
\hline ST714 & 0 & 0 & 1 \\
\hline ST889 & 0 & 1 & 0 \\
\hline K57 & 3 & 2 & 5 \\
\hline ST218 & 1 & 0 & 2 \\
\hline ST592 & 2 & 2 & 3 \\
\hline
\end{tabular}

Values given as number of patients.

patients with pneumonia, and $\sim 70 \%$ of $K$. pneumoniae isolates from the nasopharynx in adults attending otorhinolaryngology outpatient clinics. The MLST profiles further demonstrated several clones common in both pneumonia isolates and those from the nasopharynx.

Only one study has investigated a series of $K$. pneumoniae LRTIs and documented the high fatality rate $(15 / 40,37.5 \%)$ in Cambodia (Rammaert et al., 2012). The current study demonstrated community-onset pneumonia with $K$. pneumoniae had high mortality (29.7\%) despite being in a developed country with well-equipped healthcare facilities. Generally, the 30-day mortality rate of CAP is $7.3 \%$ in Asia (Song et al., 2008). The mortality rate of overall CAP is $8.3 \%$ in Taiwan (Lauderdale et al., 2005). The high mortality caused by K. pneumoniae suggested that the virulent strains played an important role in pneumonia. Our previous studies showed high mortality in bacteremic CAP caused by $K$. pneumoniae (Lin et al., 2010b). The current study demonstrated that community-onset $K$. pneumoniae pneumonia is a critical problem in the modern era in endemic areas. 
Table 4 | Comparison of microbiological characteristics among $K$. pneumoniae isolates collected from patients with community-onset pneumonia and nasopharynx from outpatients.

\begin{tabular}{|c|c|c|c|c|c|c|}
\hline Characteristic & $\begin{array}{l}\text { CAP No. } \\
(n=47)\end{array}$ & $\begin{array}{l}\text { HCAP No. } \\
(n=44)\end{array}$ & $\begin{array}{l}\text { Nasopharynx from } \\
\text { outpatients No. }(n=71)\end{array}$ & $\begin{array}{l}P \text {-value:CAP } \\
\text { vs. HCAP }\end{array}$ & $\begin{array}{l}P \text {-value: CAP } \\
\text { vs outpatient }\end{array}$ & $\begin{array}{l}P \text {-value: HCAP } \\
\text { vs outpatient }\end{array}$ \\
\hline Capsular type K1 & $13(27.7)$ & $9(20.5)$ & $14(19.7)$ & 0.422 & 0.315 & 0.924 \\
\hline Capsular type K2 & $12(25.5)$ & $9(20.5)$ & $20(28.2)$ & 0.566 & 0.752 & 0.355 \\
\hline Capsular type K1 and K2 & $25(53.2)$ & $18(40.9)$ & $34(47.9)$ & 0.241 & 0.573 & 0.465 \\
\hline Capsular type K1, K2, K5, K20, K54, and K57 & $36(76.6)$ & $25(56.8)$ & $49(69.0)$ & 0.045 & 0.369 & 0.184 \\
\hline Presence of plasmid rmpA2 & $39(83.0)$ & $24(54.5)$ & $52(73.2)$ & 0.003 & 0.233 & 0.069 \\
\hline Wild-type antibiotic susceptibility & $43(91.5)$ & 28 (63.6) & 68 (95.8) & 0.001 & 0.335 & $<0.001$ \\
\hline
\end{tabular}

Values given as number of patients (\%).

Table 5 | Factors associated with 28-day mortality in adult patients with community-acquired pneumonia (CAP) caused by K. pneumoniae.

\begin{tabular}{|c|c|c|c|}
\hline \multirow[b]{2}{*}{ Characteristic } & \multicolumn{3}{|c|}{ Univariate analysis } \\
\hline & Survival $(n=64)$ & Death $(n=27)$ & $P$-value \\
\hline Male sex & $53(82.8)$ & $23(85.2)$ & 0.781 \\
\hline \multicolumn{4}{|l|}{ Underlying diseases } \\
\hline Malignancy & $15(23.4)$ & $14(51.9)$ & 0.010 \\
\hline Neurological disorders & $18(28.1)$ & 7 (25.9) & 0.830 \\
\hline Chronic kidney disease stage $\geq 4$ & $9(14.1)$ & $4(14.8)$ & 0.925 \\
\hline Chronic lung diseases & $19(29.7)$ & $6(22.2)$ & 0.468 \\
\hline Liver cirrhosis & $1(1.6)$ & $2(7.4)$ & 0.195 \\
\hline Healthcare associated pneumonia & $29(45.3)$ & $15(55.6)$ & 0.373 \\
\hline Pneumonia Severity Index (PSI), score & $134.1 \pm 35.0$ & $167.4 \pm 39.8$ & 0.001 \\
\hline Initial presentation with septic shock & $20(31.3)$ & $15(55.6)$ & 0.032 \\
\hline Initial presentation with respiratory failure & $26(40.6)$ & $20(74.1)$ & 0.005 \\
\hline Intensive care unit admission & $35(54.7)$ & $21(77.8)$ & 0.043 \\
\hline Complicated with bacteremia & $9(14.1)$ & $3(11.1)$ & 0.704 \\
\hline Appropriate antibiotics & $57(89.7)$ & $23(85.2)$ & 0.606 \\
\hline Capsular type K1 and K2 & $31(48.4)$ & $12(44.4)$ & 0.727 \\
\hline Capsular type K1, K2, K5, K20, K54, and K57 & $43(67.2)$ & $18(66.7)$ & 0.977 \\
\hline
\end{tabular}

Values given as mean $\pm S D$ or number of patients (\%). ICU, intensive care unit.

The capsule is a major virulence factor of K. pneumoniae, and some capsular types are related to the invasive syndromes (Chung et al., 2012; Siu et al., 2012). The distribution of capsular types in each K. pneumoniae-related disease could be crucial for disease control and prevention. We determined the capsular types of $K$. pneumoniae from community-onset pneumonia. We found that capsular types K1, K2, K5, K20, K54, and K57 were prevalent in community-onset pneumonia, which have been 
reported as the major types for liver abscess in Taiwan (Fang et al., 2007). Most of the applications of MLST for K. pneumoniae were for drug-resistant strains and only a few studies have applied MLST to virulent strains (capsular type K1 or K2; Siu et al., 2011; Liao et al., 2014). In the current study, we analyzed the MLST among the virulent capsular types (K1, K2, K5, $\mathrm{K} 20$, K54, and K57) from pneumonia, and we found several ST types were prevalent in pneumonia isolates. These virulent clones and the associated increased epidemic potential may be associated with the spread in the community in endemic areas. In western countries, investigations about capsular types of $K$. pneumoniae have rarely been reported. One large-scale study conducted in Europe and North America several decades ago has found that $\mathrm{K} 2$ represented the most common types (8.9\%) among bacteremic isolates (Cryz et al., 1986). This suggest that capsular $\mathrm{K} 2$ type K. pneumoniae may be the global threat in this invasive disease.

Patients with HCAP had more exposure to healthcare environments or procedures, and the isolates may have the multidrug resistant problems. However, the virulence factors between CAP and HCAP caused by K. pneumoniae have never been compared. In this study, HCAP patients had a significantly higher Charlson Comorbidity Index and PSI score than CAP patients had. However, the number of capsular type K1, K2, $\mathrm{K} 5, \mathrm{~K} 20, \mathrm{~K} 54$, and K57, and wild-type antibiotic susceptibility strains were more common in CAP than HCAP. Overall, most of the isolates in this study showed wild-type antibiotic susceptibility. Our previous study on community-onset K. pneumonia bacteremia also displayed the prevalent wild-type antibiotic susceptibility isolates (Wu et al., 2012). Although the virulent but less drug-resistant isolates were rarely identified in western countries, they were indeed widespread in Taiwan and Asia countries. It is the remarkable features of K. pneumoniae isolates in Taiwan. These virulent capsular types are considered to be closely associated with community-acquired invasive disease or pathogenicity, and are rarely identified in nosocomial strains (Turton etal., 2010; Lin etal., 2014). We found that capsular type $\mathrm{K} 1 / \mathrm{K} 2$, traditionally known as the most virulent strains of $K$. pneumoniae types, accounted for $40 \%$ of cases of HCAP. We cannot ignore the possible risk of progression to nosocomial spread of these invasive strains, which might result in severe therapeutic problems in immunocompromised hosts.

In our previous study, we suggested that the route of entry in liver abscess is from the gastrointestinal tract (Fung et al., 2012; Lin et al., 2013). We also indicated that none of 49 patients with bacteremic CAP caused by $K$. pneumoniae showed development of $K$. pneumoniae liver abscess concomitantly (Lin et al., 2010b). The pathogenesis of pneumonia and liver abscess may be different and the gastrointestinal tract carriage may not be related to pneumonia. Pharyngeal carriage of K. pneumoniae was considered to potentially play a role in the pathogenesis of $K$. pneumoniae pneumonia in recent studies from Asia (Farida et al., 2013; Dao et al., 2014). Farida et al. have shown that the carriage rate of K. pneumoniae in healthy individuals in Semarang, Indonesia, exceeds that of S. pneumoniae in adults (Farida et al., 2013). Almost all K. pneumoniae strains have the wild-type antibiotic susceptibility to common antimicrobial agents; similar to those found in CAP patients in Semarang, Indonesia (Farida et al., 2013). In our findings, most patients from outpatient departments were healthy and had no underlying diseases. Therefore, the findings may represent the colonization status and distribution of capsular types in the general population. Consistent with the recent study conducted by Farida et al. (2013), nearly all the strains from the nasopharynx in our study showed wild-type antibiotic susceptibility. The high prevalence of virulent capsular types (K1, K2, K5, K20, K54, and $\mathrm{K} 57$ ) in the community setting may correspond to the isolates from community-onset pneumonia. The MLST profiles further demonstrated the genetic relatedness between pneumonia isolates and those from the nasopharynx. Several ST types, such as ST23, ST65, and ST86, were prevalent in both pneumonia and the nasopharynx. These findings are a first step in elucidating potential pathways leading to community-onset pneumonia with K. pneumoniae.

Our study was limited by its retrospective design. However, our data have important clinical implications because of the lack of capsular types from pneumonia and the nasopharyngeal region reported in the literature. The other limitation was that we could not explore the association between infection and colonization precisely because of the different groups studied. A future prospective study would give us the opportunity to detect colonization and infection by the same K. pneumoniae strain and the associated clinical features. Lastly, while most the patients from outpatient departments had no underlying diseases and did not receive antibiotics, they did have upper respiratory tract infections. Collecting nasopharyngeal samples from non-ill healthy adults could be the best method to investigate the nasopharyngeal carriage. Despite this limitation, we give an insight that community-onset pneumonia could have a reservoir in the nasopharynx, rather than being spread from specific sources.

In conclusion, we found a high mortality (29.7\%) among community-onset $K$. pneumoniae pneumonia. Capsular types $\mathrm{K} 1, \mathrm{~K} 2, \mathrm{~K} 5, \mathrm{~K} 20, \mathrm{~K} 54$, and $\mathrm{K} 57$ accounted for $\sim 70 \%$ of all the $K$. pneumoniae isolates from patients with pneumonia, and $\sim 70 \%$ of $K$. pneumoniae isolates from the nasopharynx in adults attending otorhinolaryngology outpatient clinics. These six capsular types and wild-type antibiotic susceptibility strains were more common in CAP than HCAP. The MLST profiles further demonstrated the genetic relatedness between most pneumonia isolates and those from the nasopharynx. The similar microbiological characteristics imply that K. pneumoniae isolates in the nasopharynx play a role in the pathogenesis of pneumonia. The distribution of capsular types in the nasopharynx might have implications for future vaccine development.

\section{AUTHOR CONTRIBUTIONS}

YTL contributed to study concept and design, and data analysis and interpretation; YTL and YPW contributed to data acquisition and manuscript drafting; FDW contributed to critical manuscript revision; and CPF contributed to the supervision of this investigation. 


\section{ACKNOWLEDGMENTS}

This study was partly supported by grants from the Ministry of Science and Technology, Taiwan, Republic of China (MOST 1032314-B-075-078-MY2), Taipei Veterans General Hospital (V103B016 and V103C-127), and Szu-Yuan Research Foundation of Internal Medicine.

\section{REFERENCES}

Chung, D. R., Park, M. H., Kim, S. H., Ko, K. S., Kang, C. I., Peck, K. R., etal. (2012). Prevalence and molecular characterization of serotype K1 Klebsiella pneumoniae strains from various clinical specimen sources in 11 Asian countries. J. Infect. 64, 622-625. doi: 10.1016/j.jinf.2012. 02.007

Cryz, S. J. Jr, Mortimer, P. M., Mansfield, V., and Germanier, R. (1986). Seroepidemiology of Klebsiella bacteremic isolates, and implications for vaccine development. J. Clin. Microbiol. 23, 687-690.

Dao, T. T., Liebenthal, D., Tran, T. K., Ngoc Thi Vu, B., Ngoc Thi Nguyen, D., Thi Tran, H. K., et al. (2014). Klebsiella pneumoniae oropharyngeal carriage in rural and urban Vietnam and the effect of alcohol consumption. PLoS ONE 9:e91999. doi: 10.1371/journal.pone.0091999

Decre, D., Verdet, C., Emirian, A., Le Gourrierec, T., Petit, J. C., Offenstadt, G., et al. (2011). Emerging severe and fatal infections due to Klebsiella pneumoniae in two university hospitals in France. J. Clin. Microbiol. 49, 3012-3014. doi: 10.1128/jcm.00676-11

Diancourt, L., Passet, V., Verhoef, J., Grimont, P. A., and Brisse, S. (2005). Multilocus sequence typing of Klebsiella pneumoniae nosocomial isolates. J. Clin. Microbiol. 43, 4178-4182. doi: 10.1128/jcm.43.8.4178-418 2.2005

Fang, C. T., Chuang, Y. P., Shun, C. T., Chang, S. C., and Wang, J. T. (2004). A novel virulence gene in Klebsiella pneumoniae strains causing primary liver abscess and septic metastatic complications. J. Exp. Med. 199, 697-705. doi: 10.1084/jem.20030857

Fang, C. T., Lai, S. Y., Yi, W. C., Hsueh, P. R., Liu, K. L., and Chang, S. C. (2007). Klebsiella pneumoniae genotype $\mathrm{K} 1$ : an emerging pathogen that causes septic ocular or central nervous system complications from pyogenic liver abscess. Clin. Infect. Dis. 45, 284-293. doi: 10.1086/519262

Farida, H., Severin, J. A., Gasem, M. H., Keuter, M., Van Den Broek, P., Hermans, P. W., et al. (2013). Nasopharyngeal carriage of Klebsiella pneumoniae and other Gram-negative bacilli in pneumonia-prone age groups in Semarang, Indonesia. J. Clin. Microbiol. 51, 1614-1616. doi: 10.1128/jcm.00 589-13

Fine, M. J., Auble, T. E., Yealy, D. M., Hanusa, B. H., Weissfeld, L. A., Singer, D. E., et al. (1997). A prediction rule to identify low-risk patients with community-acquired pneumonia. N. Engl. J. Med. 336, 243-250. doi: 10.1056/nejm1997012333 60402

Fung, C. P., Chang, F. Y., Lee, S. C., Hu, B. S., Kuo, B. I., Liu, C. Y., et al. (2002). A global emerging disease of Klebsiella pneumoniae liver abscess: is serotype $\mathrm{K} 1$ an important factor for complicated endophthalmitis? Gut 50, 420-424. doi: 10.1136/gut.50.3.420

Fung, C. P., Lin, Y. T., Lin, J. C., Chen, T. L., Yeh, K. M., Chang, F. Y., et al. (2012). Klebsiella pneumoniae in gastrointestinal tract and pyogenic liver abscess. Emerg. Infect. Dis. 18, 1322-1325. doi: 10.3201/eid1808.111053

Griffin, A. T., Wiemken, T. L., and Arnold, F. W. (2013). Risk factors for cardiovascular events in hospitalized patients with community-acquired pneumonia. Int. J. Infect. Dis. 17, e1125-e1129. doi: 10.1016/j.ijid.201 3.07.005

Hsu, C. R., Lin, T. L., Chen, Y. C., Chou, H. C., and Wang, J. T. (2011). The role of Klebsiella pneumoniae rmpA in capsular polysaccharide synthesis and virulence revisited. Microbiology 157, 3446-3457. doi: 10.1099/mic.0.05 0336-0

Lauderdale, T. L., Chang, F. Y., Ben, R. J., Yin, H. C., Ni, Y. H., Tsai, J. W., et al. (2005). Etiology of community acquired pneumonia among adult patients requiring hospitalization in Taiwan. Respir. Med. 99, 1079-1086. doi: 10.1016/j.rmed.2005.02.026

Liao, C. H., Huang, Y. T., Chang, C. Y., Hsu, H. S., and Hsueh, P. R. (2014). Capsular serotypes and multilocus sequence types of bacteremic Klebsiella pneumoniae isolates associated with different types of infections.
Eur. J. Clin. Microbiol. Infect. Dis. 33, 365-369. doi: 10.1007/s10096-01 3-1964-Z

Lin, Y. T., Chen, T. L., Siu, L. K., Hsu, S. F., and Fung, C. P. (2010a). Clinical and microbiological characteristics of community-acquired thoracic empyema or complicated parapneumonic effusion caused by Klebsiella pneumoniae in Taiwan. Eur. J. Clin. Microbiol. Infect. Dis. 29, 1003-1010. doi: 10.1007/s10096-01 0-0961-8

Lin, Y. T., Jeng, Y. Y., Chen, T. L., and Fung, C. P. (2010b). Bacteremic communityacquired pneumonia due to Klebsiella pneumoniae: clinical and microbiological characteristics in Taiwan, 2001-2008. BMC. Infect. Dis. 10:307. doi: 10.1186/14712334-10-307

Lin, Y. T., Liu, C. J., Yeh, Y. C., Chen, T. J., and Fung, C. P. (2013). Ampicillin and amoxicillin use and the risk of Klebsiella pneumoniae liver abscess in Taiwan. J. Infect. Dis. 208, 211-217. doi: 10.1093/infdis/j it 157

Lin, Y. T., Wang, F. D., Chan, Y. J., Fu, Y. C., and Fung, C. P. (2014). Clinical and microbiological characteristics of tigecycline non-susceptible Klebsiella pneumoniae bacteremia in Taiwan. BMC. Infect. Dis. 14:1. doi: 10.1186/1471-2 334-14-1

Maruyama, T., Fujisawa, T., Okuno, M., Toyoshima, H., Tsutsui, K., Maeda, H., et al. (2013). A new strategy for healthcare-associated pneumonia: a 2-year prospective multicenter cohort study using risk factors for multidrug-resistant pathogens to select initial empiric therapy. Clin. Infect. Dis. 57, 1373-1383. doi: $10.1093 / \mathrm{cid} / \mathrm{cit} 571$

Paganin, F., Lilienthal, F., Bourdin, A., Lugagne, N., Tixier, F., Genin, R., et al. (2004). Severe community-acquired pneumonia: assessment of microbial aetiology as mortality factor. Eur. Respir. J. 24, 779-785. doi: 10.1183/09031936.04.001 19503

Pan, Y. J., Lin, T. L., Chen, Y. H., Hsu, C. R., Hsieh, P. F., Wu, M. C., et al. (2013). Capsular types of Klebsiella pneumoniae revisited by wzc sequencing. PLoS ONE 8:e80670. doi: 10.1371/journal.pone.0080670

Rafat, C., Fihman, V., and Ricard, J. D. (2013). A 51-year-old man presenting with shock and lower-lobe consolidation with interlobar bulging fissure. Chest 143, 1167-1169. doi: 10.1378/chest.12-2007

Rammaert, B., Goyet, S., Beaute, J., Hem, S., Te, V., Try, P. L., et al. (2012). Klebsiella pneumoniae related community-acquired acute lower respiratory infections in Cambodia: clinical characteristics and treatment. BMC. Infect. Dis. 12:3. doi: 10.1186/1471-2334-12-3

Shon, A. S., Bajwa, R. P., and Russo, T. A. (2013). Hypervirulent (hypermucoviscous) Klebsiella pneumoniae: a new and dangerous breed. Virulence 4, 107-118. doi: 10.4161/viru. 22718

Siu, L. K., Fung, C. P., Chang, F. Y., Lee, N., Yeh, K. M., Koh, T. H., et al. (2011). Molecular typing and virulence analysis of serotype K1 Klebsiella pneumoniae strains isolated from liver abscess patients and stool samples from noninfectious subjects in Hong Kong, Singapore, and Taiwan. J. Clin. Microbiol. 49, 3761-3765. doi: 10.1128/jcm.00977-11

Siu, L. K., Yeh, K. M., Lin, J. C., Fung, C. P., and Chang, F. -Y. (2012). Klebsiella pneumoniae liver abscess: a new invasive syndrome. Lancet Infect. Dis. 12, 881887. doi: 10.1016/S1473-3099(12)70205-0

Song, J. H., Oh, W. S., Kang, C. I., Chung, D. R., Peck, K. R., Ko, K. S., et al. (2008). Epidemiology and clinical outcomes of community-acquired pneumonia in adult patients in Asian countries: a prospective study by the Asian network for surveillance of resistant pathogens. Int. J. Antimicrob. Agents 31, 107-114. doi: 10.1016/j.ijantimicag.2007.09.014

Turton, J. F., Perry, C., Elgohari, S., and Hampton, C. V. (2010). PCR characterization and typing of Klebsiella pneumoniae using capsular type-specific, variable number tandem repeat and virulence gene targets. J. Med. Microbiol. 59, 541-547. doi: 10.1099/jmm.0.015198-0

Wang, J. L., Chen, K. Y., Fang, C. T., Hsueh, P. R., Yang, P. C., and Chang, S. C. (2005). Changing bacteriology of adult community-acquired lung abscess in Taiwan: Klebsiella pneumoniae versus anaerobes. Clin. Infect. Dis. 40, 915-922. doi: 10.1086/428574

Wu, H. S., Wang, F. D., Tseng, C. P., Wu, T. H., Lin, Y. T., and Fung, C. P. (2012). Characteristics of healthcare-associated and community-acquired Klebsiella pneumoniae bacteremia in Taiwan. J. Infect. 64, 162-168. doi: 10.1016/j.jinf.2011.11.005

Yu, V. L., Hansen, D. S., Ko, W. C., Sagnimeni, A., Klugman, K. P., Von Gottberg, A., et al. (2007). Virulence characteristics of Klebsiella and clinical manifestations 
of K. pneumoniae bloodstream infections. Emerg. Infect. Dis. 13, 986-993. doi: 10.3201/eid1307.070187

Yu, W. L., Ko, W. C., Cheng, K. C., Lee, H. C., Ke, D. S., Lee, C. C., et al. (2006). Association between rmpA and magA genes and clinical syndromes caused by Klebsiella pneumoniae in Taiwan. Clin. Infect. Dis. 42, 1351-1358. doi: 10.1086/503420

Conflict of Interest Statement: The authors declare that the research was conducted in the absence of any commercial or financial relationships that could be construed as a potential conflict of interest.

Received: 25 December 2014; accepted: 30 January 2015; published online: 18 February 2015.
Citation: Lin Y-T, Wang Y-P, Wang F-D and Fung C-P (2015) Community-onset Klebsiella pneumoniae pneumonia in Taiwan: clinical features of the disease and associated microbiological characteristics of isolates from pneumonia and nasopharynx. Front. Microbiol. 6:122. doi: 10.3389/fmicb.2015.00122

This article was submitted to Infectious Diseases, a section of the journal Frontiers in Microbiology.

Copyright $\odot 2015$ Lin, Wang, Wang and Fung. This is an open-access article distributed under the terms of the Creative Commons Attribution License (CC BY). The use, distribution or reproduction in other forums is permitted, provided the original author $(s)$ or licensor are credited and that the original publication in this journal is cited, in accordance with accepted academic practice. No use, distribution or reproduction is permitted which does not comply with these terms. 